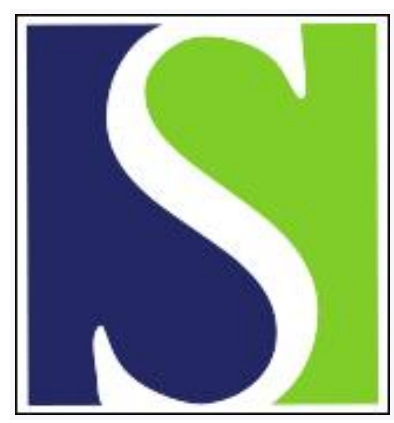

Scand J Work Environ Health 2012;38(3):228-237

https://doi.org/10.5271/sjweh.3264

Published online: 15 Dec 2011, Issue date: May 2012

Health risk behaviors and morbidity among hospital staff comparison across hospital ward medical specialties in a study of 21 Finnish hospitals

by Virtanen M, Vahtera J, Batty GD, Tuisku K, Oksanen T, Elovainio M, Ahola K, Pentti J, Salo P, Vartti A-M, Kivimäki M

Affiliation: Finnish Institute of Occupational Health, Topeliuksenkatu 41 a A, FI-00250 Helsinki, Finland. marianna.virtanen@ttl.fi

Key terms: Finland; Finland; health risk; health risk behavior; healthcare personnel; hospital; hospital staff; hospital ward; medical speciality; medication; mental health; prospective study; risk behavior; sickness absence; sickness absence

This article in PubMed: www.ncbi.nlm.nih.gov/pubmed/22173213 


\title{
Health risk behaviors and morbidity among hospital staff - comparison across hospital ward medical specialties in a study of 21 Finnish hospitals
}

\author{
by Marianna Virtanen, PhD, ${ }^{1}$ Jussi Vahtera, MD,1, 2 G David Batty, PhD, ${ }^{3}$ Katinka Tuisku, MD, ${ }^{1,4}$ Tuula Oksanen, \\ MD, ${ }^{1}$ Marko Elovainio, PhD, ${ }^{5}$ Kirsi Ahola, PhD, ${ }^{1}$ Jaana Pentti, BSc, ${ }^{1}$ Paula Salo, PhD, ${ }^{1}$ Anne-Marie Vartti, MA, \\ Mika Kivimäki, PhD, 1, 3, 8
}

Virtanen M, Vahtera J, Batty GD, Tuisku K, Oksanen T, Elovainio M, Ahola K, Pentti J, Salo P, Vartti A-M, Kivimäki M. Health risk behaviors and morbidity among hospital staff - comparison across hospital ward medical specialties in a study of 21 Finnish hospitals. Scand J Work Environ Health. 2012;38(3):228-237. doi:10.5271/sjweh.3264

Objective This study examined whether indicators of poor health and health risk behaviors among hospital staff differ between the ward specialties.

Methods Across 21 hospitals in Finland, 8003 employees (mean age 42 years, $87 \%$ women, $86 \%$ nurses) working in internal medicine, surgery, obstetrics and gynecology, pediatrics, intensive care, and psychiatry responded to a baseline survey on health and health risk behaviors (response rate 70\%). Responses were linked to records of sickness absence and medication over the following 12 months.

Results Psychiatric staff had higher odds of smoking [odds ratio (OR) 2.58, 95\% confidence interval ( $95 \% \mathrm{CI}$ ) 2.14-3.12], high alcohol use (OR 1.55, 95\% CI 1.21-1.99), physical inactivity (OR 1.30, 95\% CI 1.11-1.53), chronic physical disease (OR 1.19, 95\% CI 1.04-1.36), current or past mental disorders (OR 1.81, 95\% CI $1.50-2.17$ ), and co-occurring poor health indicators ( $\mathrm{OR} 2.65,95 \% \mathrm{CI} 2.08-3.37$ ) as compared to those working in other specialties. They also had higher odds of sickness absence due to mental disorders (OR 1.40, 95\% CI $1.02-1.92)$ and depression (OR 1.61, 95\% CI 1.02-2.55) at follow-up after adjustment for baseline health and covariates. Personnel in surgery had the lowest probability of morbidity. No major differences between specialties were found in the use of psychotropic medication.

Conclusion The prevalence of hospital employees with an adverse health risk profile is higher in psychiatric wards than other specialties.

Key terms healthcare personnel; medication; mental health; prospective study; sickness absence.

\begin{abstract}
Although studies on health inequalities between occupational groups has had important policy implications at all levels of society, recently there has been growing interest in horizontal comparisons of employees within the same organization (1). In hospital settings, there is some evidence to suggest differences in health and health behaviors between staff working in different specialties (2-21). Studies of physicians show elevated rates of "burnout", mental disorders, substance use, and suicide among psychiatrists, anesthesiologists, and phy-
\end{abstract}

sicians working in emergency or primary care although the association is not robust (2-14). In contrast, surgeons and pediatricians have reported lower rates of substance use (7), although this is not a universal finding $(9,10)$. Surgeons have also been shown to have higher (11) and lower (12) risk of death from ischemic heart disease when compared to other medical specialists. Studies of nurses suggest that psychiatric nurses report lower levels of work stress and burnout $(15,16)$ and higher levels of job autonomy (17) but also more emotional exhaustion

1 Finnish Institute of Occupational Health, Helsinki, Finland.

2 Department of Public Health, University of Turku and Turku University Hospital, Turku, Finland.

3 Department of Epidemiology and Public Health, University College London Medical School, London, UK.

4 Department of Psychiatry, Hospital District of Helsinki and Uusimaa, Helsinki, Finland.

5 Department of Service System, National Institute for Health and Welfare, Helsinki, Finland.

6 Department of Strategic and Defence Studies, National Defence University, Helsinki, Finland

7 Department of Public Health, University of Helsinki, Helsinki, Finland.

8 Institute of Behavioral Sciences, University of Helsinki, Helsinki, Finland.

Correspondence to: Dr. Marianna Virtanen, Finnish Institute of Occupational Health, Topeliuksenkatu 41 a A, FI-00250 Helsinki, Finland. [E-mail: marianna.virtanen@ttl.fi] 
(17) than their collaborators working in non-psychiatric settings. No difference was found in one study investigating suicidal behavior among psychiatric healthcare personnel and the general population (18). Other studies found nurses in non-emergency somatic medicine having higher levels of exhaustion than those working in emergency care (19), medical care nurses reporting higher workload than geriatric care nurses (20), and intensive care nursing staff having particularly high burnout rates (21). A major limitation of most studies in this field, however, is a reliance on small sample sizes and, with few exceptions $(3,5,11,12)$, exclusive use of cross-sectional self-report data. In addition, a broad range of health risks and their accumulation has not previously been investigated.

In this study of 8003 Finnish public hospital employees, we examined the association of medical specialty with employee lifestyle and morbidity. We also examined the onset of sickness absence and medication use by using record linkage to national health registers which has not, to our knowledge, previously been examined. Based on earlier evidence, we anticipated that employees working in psychiatry may have more health problems than those in other medical domains.

\section{Methods}

\section{Sample and procedure}

Twenty-one hospitals in six Finnish hospital districts participate in the ongoing Work and Health in Finnish Hospital Personnel study (22). In Finland, there are 21 hospital districts, and the study hospitals provide specialized healthcare for approximately $36 \%$ of the Finnish population. The study is coordinated by the Finnish Institute of Occupational Health and approved by the ethics committees of the Finnish Institute of Occupational Health, the Hospital District of Helsinki and Uusimaa, and the Social Insurance Institute (SII) of Finland.

Eligible employees comprised medical staff $(\mathrm{N}=13229)$ who worked in any of the 14 medical specialty services provided by the participating hospitals. According to national registers, 396 either retired or died during the follow-up, resulting in 12833 eligible employees. In 2004, a questionnaire was mailed to all these employees. Of the $8971(70 \%)$ who responded to the survey, $8003(89 \%)$ had information on the type of employment contract; these individuals comprised the analytic cohort. Each survey respondent was then linked to employers' registry data on demographic factors and hospital characteristics and separately to individual records of the nationwide health registers.

\section{Measures}

Data on age, gender, occupation (further categorized as physicians/other professionals, nurses, practical/ assistant nurses), type of employment (permanent/ temporary), hospital district, ward type (inpatient bed ward versus outpatient clinic), and work unit medical specialty were obtained from the employers' registers. Participating hospitals encompass the following 13 specialties: internal medicine, surgery, obstetrics and gynecology, pediatrics, intensive care, psychiatry, pulmonary diseases, ophthalmology, otology, neurology, dermatology and venereology, oncology, and physiatry. Due to the relatively low number of employees within each specialty, pulmonary diseases, ophthalmology, otology, neurology, dermatology and venereology, oncology, and physiatry were unified into "other somatic diseases".

Data on marital status (married/co-habiting versus not), health-risk behaviors, and work characteristics were based on survey responses. Health risk behaviors included smoking status (current smoker versus nonsmoker), high alcohol consumption (average weekly consumption $\geq 210 \mathrm{~g}$ of absolute alcohol) (23), physical inactivity $[<2.0$ metabolic equivalent task (MET) hours per day, corresponding to approximately 30 minutes of walking] (24), and obesity [body mass index (BMI) $\geq 30 \mathrm{~kg} / \mathrm{m}^{2}$ ]. Work characteristics included self-reported assessment of work stress using measures of the major work stress models [ie, job strain as expressed by the demand-control model (25), effort-reward imbalance (26), and organizational justice (27)]. The items were continuous ranging from $1-5$. In addition, exposure to violence at work was assessed by questions of frequency and type of violence experiences (28). Frequent or severe violence was identified if the respondent reported physical or mental violence or violence towards ward property occurring at least monthly, or ever being exposed to violence with a weapon.

Information on diagnosed chronic physical disease was derived from (i) survey responses (respondents indicated if they had ever had a physician-diagnosis of a range of 15 common chronic somatic diseases: allergy, asthma, chronic bronchitis, hypertension, myocardial infarction, angina, ischemic attack, arthrosis, rheumatoid arthritis, fibromyalgia, sciatica, gastric ulcer, migraine, diabetes mellitus, and cancer) (29); (ii) the SII's special reimbursement register (at the end of the survey year, the following were identified: hypertension, cardiac failure, ischemic heart disease, diabetes, asthma or other chronic obstructive lung disease, and rheumatoid arthritis); and (iii) the Finnish Cancer Registry (information on cancer diagnosed during the survey year or four preceding years).

Information on current or past diagnosed mental disorders was based on the survey response (whether a doctor had ever diagnosed the participant as suffering 
from a mental disorder), SII register data on psychiatric sickness absence during the survey year [International Classification of Diseases (ICD), $10^{\text {th }}$ Revision, ICD10 F-diagnoses], state-subsidized psychotherapy, or purchased antidepressant medication (World Health Organization's Anatomical Therapeutic Chemical ATC code N06A) (30) during the survey year.

At follow-up, the participants' sickness absence records and medication use were assessed in 2005, ie, the year following the survey and derived from the SII records. In order to assess new-onset morbidity, presence of sickness absence, or medication use during the survey year (in 2004) was adjusted for in the analyses. Data included all granted sickness absence periods of $>9$-day duration with the associated ICD-10 diagnostic code. We grouped the diagnoses of sickness absence into mental disorders (F-codes) and physical illnesses (other codes), depressive disorders (F32-F34), and musculoskeletal disorders (M50-M54). Medication use included data on purchased antidepressant medication (ATC code N06A), anxiolytics and hypnotics (N05B, N05C), and pain medicine (M01A, N02). Personal identification numbers, a unique number assigned to all citizens in Finland, were used to link all study participants to comprehensive national prescription and health registers.

\section{Statistical analysis}

For binary outcomes (health behaviors, morbidity, and medication use), we used binary logistic regression analysis and expressed the results as odds ratios (OR) and their $95 \%$ confidence intervals (95\% CI). Employees in each specialty were compared to the participants in other specialties, thus, the reference group varied in each comparison. Adjustments were made for demographics, hospital, work unit, and work characteristics. In health outcomes, we also adjusted the models for health risk behaviors and, in the longitudinal analyses, for baseline health/medication use. We constructed an additional outcome variable indicating the number of co-occurring health problems at baseline: smoking, high alcohol use, obesity, physical inactivity, somatic disease (diagnosed chronic disease or somatic sickness absence), and mental disorder (diagnosed mental disorders, psychiatric sickness absence, or antidepressant medication use). This variable ranged from $0-6$ and was recoded into categories of $0,1,2$, or $\geq 3$ health problems. Multinomial regression analysis was performed to compare each specialty against all other specialties regarding the probability of the number of health problems present: 1 versus 0,2 versus 0 and $\geq 3$ versus 0 health problems. In the prospective part of the study, we used binary logistic regression analysis to examine the association of specialty with sickness absence or medication use in 2005, after adjustment for sickness absence and medi- cation use in 2004. All analyses were performed using the SAS statistical software, version 9.2 (SAS Institute, Inc, Cary, NC, USA).

\section{Results}

The mean age of the participants was 42.2 [standard deviation (SD)8.8, range 20-63] years. Employees in intensive care units were slightly younger than the others (mean age 40 years; data not shown). Men were overrepresented in the psychiatric units and under-represented in internal medicine, obstetrics and gynecology, and pediatrics (table 1). Practical nurses worked more often in psychiatry and less often in intensive care units whereas temporary employment contracts were more common in pediatrics and less common among employees working in "other somatic disease specialties". Outpatient care was more common in surgery and "other specialties".

\section{Baseline work characteristics, lifestyle, and health}

Regarding work characteristics, employees in psychiatry and obstetrics and gynecology perceived lower job strain than the others while employees in internal medicine and surgery reported higher job strain $(\mathrm{P}<0.0001)$. Employees in obstetrics \& gynecology, pediatrics, and psychiatry had the lowest effort-reward imbalance scores whereas employees in intensive care had higher scores than the others $(\mathrm{P}<0.0001)$. Staff in psychiatry and internal medicine reported higher organizational justice, an indicator of the quality of leadership at the workplace, while employees in intensive care, surgery and "other somatic diseases" reported lower levels of justice than the rest of the participants $(\mathrm{P}<0.0001)$. However, employees working in psychiatry were exposed to frequent or severe violence in their work: the lowest exposure to violence was observed in obstetrics \& gynecology (4\%) and pediatrics (7\%) while in psychiatry the corresponding prevalence was $53 \%(\mathrm{P}<0.0001$; data not shown).

We found a higher prevalence of smoking among employees in the fields of intensive care $(15 \%)$ and psychiatry (21\%) compared with employees in other specialties (table 2). The corresponding OR was 1.35 in intensive care, while in psychiatry it was 2.58 after adjustment for covariates. Lower smoking prevalence was found in obstetrics and gynecology $(6 \%$, OR 0.56$)$ and pediatrics $(6 \%$, OR 0.50$)$. Higher probability of excess alcohol consumption (9\%, OR 1.55) and physical inactivity ( $20 \%$, OR 1.30$)$ was found among employees working in psychiatry whereas lower probability of obesity was found in surgery $(8 \%$, OR 0.79$)$. Results were largely replicated when we restricted the analyses to nurses only. Novel findings were that nurses in surgery 
Table 1. Baseline characteristics of the study population of hospital employees by hospital ward medical specialty.

\begin{tabular}{|c|c|c|c|c|c|c|c|c|c|c|c|c|c|c|c|c|c|}
\hline \multirow[t]{2}{*}{ Characteristic } & \multicolumn{2}{|c|}{$\begin{array}{c}\text { All } \\
(\mathrm{N}=8003)\end{array}$} & \multicolumn{2}{|c|}{$\begin{array}{c}\text { Internal } \\
\text { medicine } \\
(\mathrm{N}=1147)\end{array}$} & \multicolumn{2}{|c|}{$\begin{array}{l}\text { Surgery } \\
(N=1769)\end{array}$} & \multicolumn{2}{|c|}{$\begin{array}{l}\text { Obstetrics \& } \\
\text { Gynecology } \\
(\mathrm{N}=665)\end{array}$} & \multicolumn{2}{|c|}{$\begin{array}{l}\text { Pediatrics } \\
(\mathrm{N}=702)\end{array}$} & \multicolumn{2}{|c|}{$\begin{array}{c}\text { Intensive } \\
\text { care }(\mathrm{N}=538)\end{array}$} & \multicolumn{2}{|c|}{$\begin{array}{l}\text { Other somatic } \\
\text { disease } \\
\text { specialties }{ }^{a} \\
(\mathrm{~N}=1607) \\
\end{array}$} & \multicolumn{2}{|c|}{$\begin{array}{c}\text { Psychiatry } \\
(\mathrm{N}=1575)\end{array}$} & \multirow[t]{2}{*}{ P-value ${ }^{b}$} \\
\hline & $\mathrm{N}$ & $\%$ & $\mathrm{~N}$ & $\%$ & $\mathrm{~N}$ & $\%$ & $\mathrm{~N}$ & $\%$ & $\mathrm{~N}$ & $\%$ & $\mathrm{~N}$ & $\%$ & $\mathrm{~N}$ & $\%$ & $\mathrm{~N}$ & $\%$ & \\
\hline Gender & & & & & & & & & & & & & & & & & $<0.0001$ \\
\hline Women & 7000 & 87 & 1055 & 92 & 1575 & 89 & 644 & 97 & 667 & 95 & 457 & 85 & 1445 & 90 & 1157 & 73 & \\
\hline Men & 1003 & 13 & 92 & 8 & 194 & 11 & 21 & 3 & 35 & 5 & 81 & 15 & 162 & 10 & 418 & 27 & \\
\hline Occupation & & & & & & & & & & & & & & & & & $<0.0001$ \\
\hline Physician / specialist & 1115 & 14 & 132 & 12 & 199 & 11 & 62 & 9 & 88 & 13 & 35 & 7 & 301 & 19 & 298 & 19 & \\
\hline Nurse & 5597 & 70 & 801 & 70 & 1344 & 76 & 518 & 78 & 528 & 75 & 473 & 88 & 1091 & 68 & 842 & 53 & \\
\hline Practical nurse & 1291 & 16 & 214 & 19 & 226 & 13 & 85 & 13 & 86 & 12 & 30 & 6 & 215 & 13 & 435 & 28 & \\
\hline Type of employment & & & & & & & & & & & & & & & & & 0.003 \\
\hline Permanent & 6144 & 77 & 860 & 75 & 1376 & 78 & 510 & 77 & 503 & 72 & 402 & 75 & 1269 & 79 & 1224 & 78 & \\
\hline Temporary & 1859 & 23 & 287 & 25 & 393 & 22 & 155 & 23 & 199 & 28 & 136 & 25 & 338 & 21 & 351 & 22 & \\
\hline Marital status & & & & & & & & & & & & & & & & & 0.30 \\
\hline Married / co-habiting & 6222 & 78 & 890 & 78 & 1374 & 78 & 521 & 78 & 530 & 76 & 415 & 77 & 1283 & 80 & 1209 & 77 & \\
\hline $\begin{array}{l}\text { Non-married / } \\
\text {-co-habiting }\end{array}$ & 1781 & 22 & 257 & 22 & 395 & 22 & 144 & 22 & 172 & 25 & 123 & 23 & 324 & 20 & 366 & 23 & \\
\hline Ward type & & & & & & & & & & & & & & & & & $<0.0001$ \\
\hline Inpatient bed ward & 4999 & 62 & 855 & 75 & 953 & 54 & 470 & 71 & 540 & 77 & 538 & 100 & 556 & 35 & 1087 & 69 & \\
\hline Outpatient clinic & 3004 & 38 & 292 & 25 & 816 & 46 & 195 & 29 & 162 & 23 & 0 & 0 & 1051 & 65 & 488 & 31 & \\
\hline
\end{tabular}

a Pulmonary diseases, ophthalmology, otology, neurology, dermatology \& venereology, oncology, and physiatry.

${ }^{\mathrm{b}} \mathrm{P}$-value for heterogeneity.

Table 2. Association between hospital ward medical specialty and health risk behaviors among hospital staff at baseline. [OR=0dds ratio; $95 \% \mathrm{Cl}=95 \%$ confidence interval]

\begin{tabular}{|c|c|c|c|c|c|c|c|c|c|c|c|c|c|c|c|c|}
\hline & \multicolumn{4}{|c|}{ Smoking } & \multicolumn{4}{|c|}{ High alcohol consumption } & \multicolumn{4}{|c|}{ Obesity } & \multicolumn{4}{|c|}{ Physical inactivity } \\
\hline & $\mathrm{N}$ & $\%$ & $\mathrm{OR}^{\mathrm{a}}$ & $95 \% \mathrm{Cl}^{\text {a }}$ & $\mathrm{N}$ & $\%$ & $\mathrm{OR}^{\mathrm{a}}$ & $95 \% \mathrm{Cl}^{\text {a }}$ & $\mathrm{N}$ & $\%$ & $\mathrm{OR}^{\mathrm{a}}$ & $95 \% \mathrm{Cl}^{\mathrm{a}}$ & $\mathrm{N}$ & $\%$ & $\mathrm{OR}^{\mathrm{a}}$ & $95 \% \mathrm{Cl}^{\text {a }}$ \\
\hline \multicolumn{17}{|l|}{$\begin{array}{l}\text { Specialty, total } \\
\text { staff }(N=8003)\end{array}$} \\
\hline $\begin{array}{l}\text { Internal medicine } \\
\text { vs others }{ }^{b}\end{array}$ & 118 & 10 & 0.97 & $0.78-1.21$ & 58 & 5 & 1.00 & $0.74-1.34$ & 108 & 9 & 0.87 & $0.69-1.08$ & 218 & 19 & 1.06 & $0.90-1.26$ \\
\hline $\begin{array}{l}\text { Surgery vs } \\
\text { others }^{b}\end{array}$ & 199 & 11 & 1.19 & $0.99-1.44$ & 84 & 5 & 0.81 & $0.62-1.05$ & 143 & 8 & 0.79 & $0.65-0.97$ & 295 & 17 & 0.93 & $0.80-1.08$ \\
\hline $\begin{array}{l}\text { Obstetrics and } \\
\text { gynecology vs } \\
\text { others }^{b}\end{array}$ & 40 & 6 & 0.56 & $0.40-0.78$ & 31 & 5 & 1.03 & $0.70-1.52$ & 71 & 11 & 1.13 & $0.86-1.47$ & 112 & 17 & 0.91 & $0.73-1.13$ \\
\hline $\begin{array}{l}\text { Pediatrics vs } \\
\text { others }^{b}\end{array}$ & 43 & 6 & 0.50 & $0.36-0.70$ & 29 & 4 & 0.90 & $0.60-1.34$ & 72 & 10 & 1.10 & $0.84-1.43$ & 125 & 18 & 0.98 & $0.79-1.20$ \\
\hline $\begin{array}{l}\text { Intensive care } \\
\text { vs others }{ }^{b}\end{array}$ & 83 & 15 & 1.35 & $1.03-1.76$ & 31 & 6 & 1.14 & $0.76-1.72$ & 58 & 11 & 1.08 & $0.80-1.45$ & 83 & 15 & 0.79 & $0.61-1.02$ \\
\hline $\begin{array}{l}\text { Other somatic } \\
\text { diseases }{ }^{c} \text { vs } \\
\text { others }^{b}\end{array}$ & 130 & 8 & 0.88 & $0.71-1.09$ & 73 & 5 & 0.75 & $0.57-1.00$ & 140 & 9 & 0.93 & $0.76-1.15$ & 301 & 19 & 1.10 & $0.94-1.28$ \\
\hline $\begin{array}{l}\text { Psychiatry vs } \\
\text { others }^{b}\end{array}$ & 329 & 21 & 2.58 & $2.14-3.12$ & 144 & 9 & 1.55 & $1.21-1.99$ & 200 & 13 & 1.17 & $0.95-1.43$ & 322 & 20 & 1.30 & $1.11-1.53$ \\
\hline \multicolumn{17}{|l|}{$\begin{array}{l}\text { Specialty, nurses } \\
(\mathrm{N}=6888)\end{array}$} \\
\hline $\begin{array}{l}\text { Internal medicine } \\
\text { vs others }{ }^{b}\end{array}$ & 111 & 11 & 0.97 & $0.78-1.22$ & 43 & 4 & 1.01 & $0.71-1.42$ & 101 & 10 & 0.90 & $0.72-1.14$ & 193 & 19 & 1.11 & $0.93-1.32$ \\
\hline $\begin{array}{l}\text { Surgery vs } \\
\text { others }^{b}\end{array}$ & 182 & 12 & 1.16 & $0.96-1.41$ & 51 & 3 & 0.69 & $0.50-0.95$ & 128 & 8 & 0.78 & $0.64-0.97$ & 254 & 16 & 0.92 & $0.79-1.09$ \\
\hline $\begin{array}{l}\text { Obstetrics and } \\
\text { gynecology } \\
\text { vs others }{ }^{b}\end{array}$ & 34 & 6 & 0.48 & $0.34-0.69$ & 21 & 3 & 0.93 & $0.58-1.48$ & 66 & 11 & 1.12 & $0.85-1.48$ & 100 & 17 & 0.90 & $0.71-1.13$ \\
\hline $\begin{array}{l}\text { Pediatrics vs } \\
\text { others }^{b}\end{array}$ & 41 & 7 & 0.51 & $0.36-0.72$ & 24 & 4 & 1.04 & $0.67-1.61$ & 66 & 11 & 1.10 & $0.84-1.46$ & 113 & 18 & 1.03 & $0.83-1.29$ \\
\hline $\begin{array}{l}\text { Intensive care vs } \\
\text { others }^{\mathrm{b}}\end{array}$ & 81 & 16 & 1.40 & $1.06-1.84$ & 27 & 5 & 1.28 & $0.82-1.97$ & 51 & 10 & 0.99 & $0.72-1.36$ & 76 & 15 & 0.77 & $0.59-1.00$ \\
\hline $\begin{array}{l}\text { Other somatic } \\
\text { diseases }^{c} \text { vs } \\
\text { others }^{b}\end{array}$ & 118 & 9 & 0.91 & $0.73-1.15$ & 45 & 3 & 0.70 & $0.49-1.00$ & 122 & 9 & 0.96 & $0.77-1.20$ & 234 & 18 & 1.08 & $0.91-1.28$ \\
\hline $\begin{array}{l}\text { Psychiatry vs } \\
\text { others }^{b}\end{array}$ & 308 & 24 & 2.80 & $2.29-3.43$ & 109 & 9 & 1.68 & $1.23-2.29$ & 174 & 14 & 1.19 & $0.95-1.49$ & 252 & 20 & 1.27 & $1.05-1.53$ \\
\hline
\end{tabular}


and "other somatic diseases" had a lower probability of high alcohol consumption (3\%, OR 0.69, and 3\%, OR 0.70 , respectively) than nurses in other specialties.

At baseline, psychiatric staff had higher odds of diagnosed chronic physical disease (64\%, OR 1.18) and diagnosed mental disorder (18\%, OR 1.79) when compared to other specialties combined (table 3.). Lower probability of physical disease was found for employees working in obstetrics \& gynecology $(57 \%$, OR 0.85 ), and lower probability for mental disorder was found for those working in surgery $(10 \%$, OR 0.79$)$. Adjustment for health risk behaviors, mental or physical morbidity, and work characteristics had little effect on the associations except for the lower odds of physical disease in obstetrics and gynecology which attenuated after adjustment. A sub-group analysis among nurses revealed largely similar relationships to those found for the total cohort.

As seen in figure 1, co-occurring health problems were more common among psychiatric staff than employees in non-psychiatric specialties. For the former, the fully adjusted OR of having 1 risk factor versus 0 was 1.41 (95\% CI $1.18-1.69)$, 2 versus 0 was 2.14 (95\% CI $1.77-2.60)$, and $\geq 3$ versus 0 was 2.65 (95\% CI 2.08-3.37), as compared with those in non-psychiatric specialties. Of the other medical specialties, the odds of having 1 indicator versus 0 was slightly elevated (OR $1.31,95 \%$ CI $1.02-1.68)$ only in intensive care. In con- trast, surgical staff had lower probabilities of having 1 , 2 , or 3 indicators of poor health, and the OR decreased as the number of co-occurring indicators increased. Of employees in psychiatry, $15 \%$ had $\geq 3$ risk indicators co-occurring while the corresponding proportions in surgery, obstetrics \& gynecology, and pediatrics were $8 \%, 7 \%$, and $7 \%$, respectively (data not shown). In psychiatry, $15 \%$ of the staff had no health problems at all whereas the corresponding figures in surgery, obstetrics $\&$ gynecology, and pediatrics were $25 \%, 25 \%$, and $26 \%$, respectively.

\section{Morbidity and medication use at follow-up}

During the 1-year follow-up period, $18 \%$ of the participants had somatic sickness absence, $4 \%$ had any psychiatric sickness absence, $2 \%$ had sickness absence due to depression, $3 \%$ had sickness absence due to musculoskeletal disorders, $6 \%$ had antidepressant treatment, $5 \%$ had anxiolytic or hypnotic medication, and $16 \%$ had prescribed pain medicine use. Analyses carried out after adjustment for relevant covariates and baseline physical and mental health indicators revealed no association between medical specialty and sickness absence due to physical illnesses (table 4) or musculoskeletal disorders (data not shown). However, sickness absence due to mental disorders was more likely among psychiatric staff (OR 1.40 for sickness absence due to all-cause

Table 3. Association between hospital ward medical specialty and health indicators among hospital staff at baseline. [OR=0dds ratio; $95 \% \mathrm{Cl}=95 \%$ confidence interval].

\begin{tabular}{|c|c|c|c|c|c|c|c|c|c|c|c|c|}
\hline & \multicolumn{6}{|c|}{ Diagnosed chronic physical disease } & \multicolumn{6}{|c|}{ Current or past diagnosed mental disorder } \\
\hline & $\mathrm{N}$ & $\%$ & $\mathrm{OR}^{\mathrm{a}}$ & $95 \% \mathrm{Cl}^{\text {a }}$ & $\mathrm{OR}{ }^{\mathrm{b}}$ & $95 \% \mathrm{Cl}^{\mathrm{b}}$ & $\mathrm{N}$ & $\%$ & $\mathrm{OR}{ }^{\mathrm{a}}$ & $95 \% \mathrm{Cl}^{\text {a }}$ & $\mathrm{OR}{ }^{\mathrm{b}}$ & $95 \% \mathrm{Cl}^{\mathrm{b}}$ \\
\hline \multicolumn{13}{|l|}{ Specialty, total staff ( $N=7779)$} \\
\hline Internal medicine vs others ${ }^{c}$ & 674 & 60 & 0.98 & $0.86-1.12$ & 0.96 & $0.84-1.10$ & 130 & 12 & 0.95 & $0.77-1.16$ & 0.93 & $0.76-1.15$ \\
\hline Surgery vs others ${ }^{c}$ & 1025 & 59 & 0.96 & $0.86-1.08$ & 0.96 & $0.85-1.08$ & 172 & 10 & 0.79 & $0.66-0.95$ & 0.77 & $0.64-0.92$ \\
\hline $\begin{array}{l}\text { Obstetrics and gynecology vs } \\
\text { others }{ }^{c}\end{array}$ & 368 & 57 & 0.85 & $0.72-1.00$ & 0.86 & $0.73-1.02$ & 72 & 11 & 0.88 & $0.68-1.15$ & 0.94 & $0.72-1.22$ \\
\hline Pediatrics vs others ${ }^{c}$ & 407 & 59 & 0.98 & $0.83-1.16$ & 1.00 & $0.85-1.18$ & 69 & 10 & 0.79 & $0.60-1.02$ & 0.82 & $0.63-1.08$ \\
\hline Intensive care vs others ${ }^{c}$ & 317 & 61 & 1.13 & $0.93-1.37$ & 1.12 & $0.92-1.36$ & 58 & 11 & 0.97 & $0.72-1.31$ & 0.90 & $0.67-1.22$ \\
\hline $\begin{array}{l}\text { Other somatic diseases }{ }^{d} \\
\text { vs others }{ }^{c}\end{array}$ & 935 & 60 & 0.96 & $0.85-1.09$ & 0.95 & $0.84-1.08$ & 201 & 13 & 1.10 & $0.92-1.33$ & 1.11 & $0.92-1.34$ \\
\hline Psychiatry vs others ${ }^{c}$ & 974 & 64 & 1.18 & $1.04-1.33$ & 1.19 & $1.04-1.36$ & 274 & 18 & 1.79 & $1.52-2.12$ & 1.81 & $1.50-2.17$ \\
\hline \multicolumn{13}{|l|}{ Specialty, nurses ( $N=6689$ ) } \\
\hline Internal medicine vs others ${ }^{c}$ & 610 & 61 & 1.04 & $0.90-1.20$ & 1.03 & $0.89-1.19$ & 116 & 12 & 0.94 & $0.75-1.16$ & 0.90 & $0.72-1.13$ \\
\hline Surgery vs others ${ }^{c}$ & 912 & 60 & 0.97 & $0.86-1.10$ & 0.96 & $0.85-1.09$ & 159 & 10 & 0.81 & $0.67-0.98$ & 0.77 & $0.63-0.94$ \\
\hline $\begin{array}{l}\text { Obstetrics and gynecology } \\
\text { vs others }{ }^{c}\end{array}$ & 328 & 56 & 0.82 & $0.69-0.98$ & 0.83 & $0.70-1.00$ & 68 & 12 & 0.93 & $0.71-1.22$ & 1.01 & $0.76-1.33$ \\
\hline Pediatrics vs others ${ }^{c}$ & 350 & 58 & 0.95 & $0.80-1.13$ & 0.96 & $0.80-1.15$ & 64 & 11 & 0.85 & $0.65-1.12$ & 0.91 & $0.69-1.21$ \\
\hline Intensive care vs others ${ }^{c}$ & 295 & 60 & 1.11 & $0.91-1.36$ & 1.11 & $0.91-1.35$ & 54 & 11 & 0.96 & $0.71-1.31$ & 0.91 & $0.67-1.24$ \\
\hline $\begin{array}{l}\text { Other somatic diseases }{ }^{d} \\
\text { vs others }^{c}\end{array}$ & 772 & 61 & 0.98 & $0.86-1.13$ & 0.97 & $0.84-1.11$ & 170 & 13 & 1.13 & $0.92-1.38$ & 1.11 & $0.91-1.36$ \\
\hline Psychiatry vs others ${ }^{c}$ & 787 & 64 & 1.15 & $1.00-1.32$ & 1.18 & $1.01-1.38$ & 205 & 17 & 1.53 & $1.26-1.86$ & 1.53 & $1.24-1.90$ \\
\hline
\end{tabular}

a Model I: adjusted for age, gender, occupation, type of employment, marital status, ward type, and hospital district.

${ }^{\mathrm{b}}$ Model II: Model I + adjustment for smoking, alcohol use, physical activity, body mass index, mental /physical morbidity, and work characteristics.

c Others: all other specialties combined (=reference group).

d Pulmonary diseases, ophthalmology, otology, neurology, dermatology \& venereology, oncology, and physiatry. 


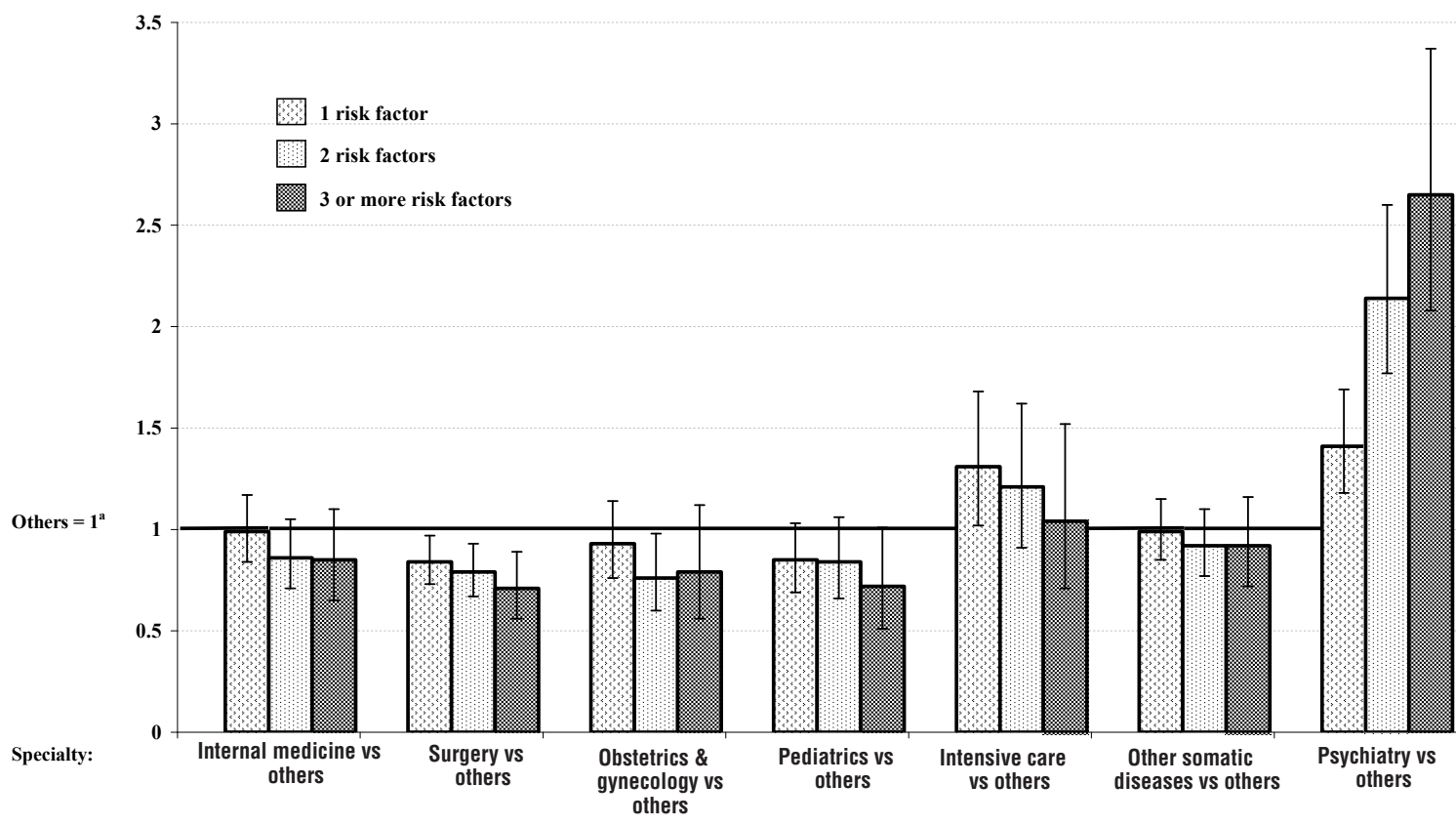

Figure 1. Comparison between each hospital ward specialty with all other specialties combined, regarding the probability of employees having 1 , 2 , or $\geq 3$ indicators of poor health versus 0 indicators at baseline. Models are adjusted for age, gender, occupation, type of employment, marital status, ward type, hospital district, and work characteristics. ${ }^{a}$ Others: all other specialities combined (=reference group)

Table 4. Association of hospital ward medical specialty with sickness absence and medication use among hospital staff at follow-up. [OR=odds ratio; $95 \% \mathrm{Cl}=95 \%$ confidence interval].

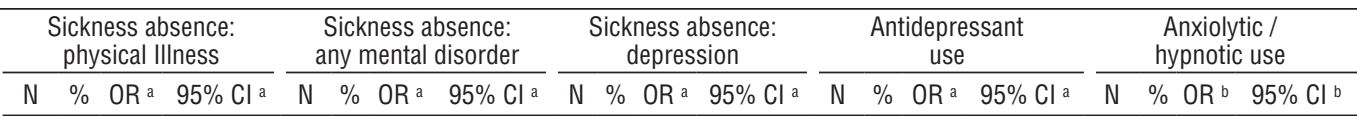

Specialty, total staff

$(\mathrm{N}=7779)$

Internal medicine vs others ${ }^{d}$

Surgery vs others ${ }^{d}$ Obstetrics and gynecology vs others ${ }^{d}$

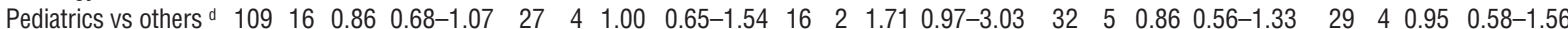
Intensive care vs others ${ }^{d}$

Other somatic diseases ${ }^{c}$ vs others ${ }^{d}$

Psychiatry vs others ${ }^{d}$

Specialty, nurses

( $N=6689$ )

Internal medicine vs others ${ }^{d}$

Surgery vs others ${ }^{d}$ Obstetrics and gynecology vs others ${ }^{d}$

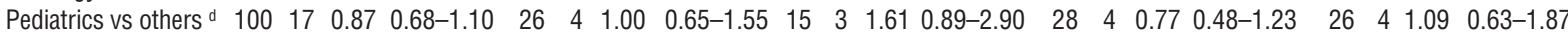
$\begin{array}{llllllllllllllllllllll}\text { Intensive care } & 95 & 19 & 1.00 & 0.77-1.29 & 26 & 5 & 1.08 & 0.69-1.69 & 10 & 2 & 1.18 & 0.59-2.40 & 23 & 5 & 0.74 & 0.44-1.24 & 16 & 3 & 0.57 & 0.28-1.15\end{array}$ vs others ${ }^{d}$

Other somatic diseases ${ }^{b}$ vs others ${ }^{d}$ Psychiatry vs others $^{d}$

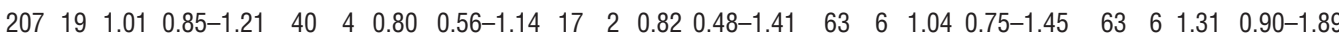

$\begin{array}{llllllllllllllllllll}325 & 19 & 1.12 & 0.96-1.30 & 56 & 3 & 0.78 & 0.57-1.07 & 16 & 1 & 0.46 & 0.27-0.81 & 80 & 5 & 0.89 & 0.66-1.21 & 64 & 4 & 0.75 & 0.52-1.07\end{array}$ $\begin{array}{lllllllllllllllllllll}125 & 19 & 1.20 & 0.96-1.49 & 23 & 4 & 0.89 & 0.56-1.40 & 8 & 1 & 0.72 & 0.34-1.53 & 40 & 6 & 1.22 & 0.82-1.83 & 40 & 6 & 1.53 & 0.96-2.42\end{array}$

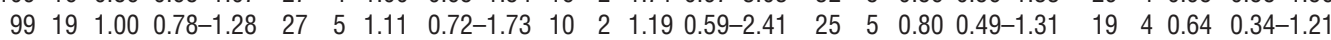

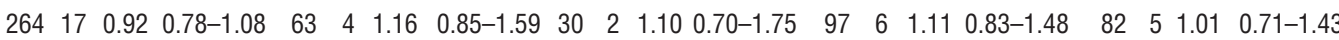

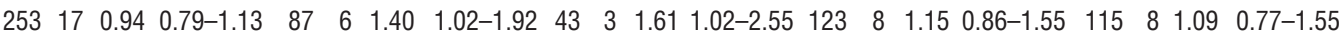

$19720 \quad 1.06 \quad 0.88-1.27 \quad 38 \quad 4 \quad 0.79 \quad 0.55-1.14 \quad 16 \quad 2 \quad 0.81 \quad 0.46-1.42 \quad 57 \quad 6 \quad 1.07 \quad 0.75-1.52 \quad 46 \quad 5 \quad 1.10 \quad 0.71-1.69$

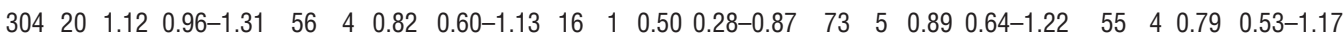

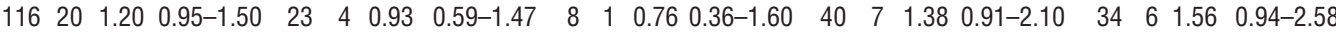

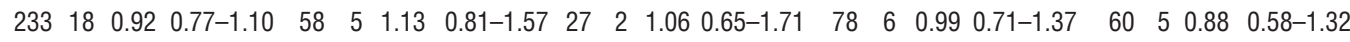

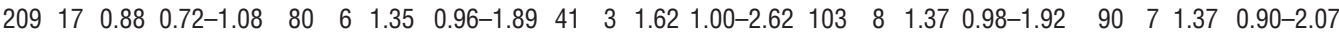

${ }^{a}$ Adjusted for baseline age, gender, occupation, type of employment, marital status, ward type, hospital district, smoking, alcohol use, physical activity, body mass index, mental and physical morbidity, antidepressant use, and work characteristics.

${ }^{\mathrm{b}}$ Adjusted as above and additionally for baseline anxiolytic/hypnotic use.

c Pulmonary diseases, ophthalmology, otology, neurology, dermatology and venereology, oncology, and physiatry.

${ }^{\mathrm{d}}$ Others: all other specialties combined (=reference group). 
mental disorders and 1.61 for depression) than among those working in other specialties. Employees in surgery had lower odds of sickness absence due to depression (OR 0.46). The results were quite similar when restricted to nurses. No major differences were found in relation to the use of psychotropic medication after adjustment for baseline mental health and medication use (data on pain medication not shown). We performed sensitivity analyses excluding employees with temporary job contracts. The results were largely replicated (available upon request).

\section{Trends in antidepressant use}

We sought to assess the question of health-related selection (that is, whether psychiatric staff already had higher morbidity a long time before the study year) by examining the prevalence of yearly antidepressant use from 1995-2005 and comparing psychiatric staff with nonpsychiatric staff (data not shown). We found that until 1998, there was no significant difference in recorded antidepressant use between staff in psychiatric and nonpsychiatric settings ( $\mathrm{P}$-values $\geq 0.06$, OR ranging from 1.3-1.4). Since 1998, a significant difference was found in every year of follow-up with higher rates of medication use among those working in psychiatry (P-values $\leq 0.006$, OR ranging from 1.4-1.8).

\section{Discussion}

This study of 8003 hospital employees showed a striking difference between psychiatry and other specialties in the prevalence of employees with an adverse health risk profile. Co-occurrence of health problems was also more prevalent among psychiatric personnel: the odds of having $\geq 3$ indicators of health problems was 2.65 -fold among psychiatric staff compared with non-psychiatric staff. Thus, various risk factors for morbidity and mortality (such as smoking, high alcohol use, physical inactivity, chronic somatic diseases, and mental disorders) seem to cluster among psychiatric hospital staff.

This study is apparently the first to examine prospectively differences between medical specialties in the onset of diagnosis-specific sickness absence and medication use among staff. After adjustment for baseline health, medication and covariates, we found no association of working in a specific medical specialty with subsequent sickness absence due to physical illnesses, musculoskeletal disorders, anxiolytic or hypnotic use, or pain medication use. However, sickness absence (with the diagnoses of mental disorders in general and due to depression in particular) were 1.4-1.6 times more likely to occur among psychiatric staff compared to personnel in other specialties. In contrast, surgical staff had lower likelihood of sickness absence due to depression.

Our findings are in agreement with earlier mainly cross-sectional studies focused on specialized physicians, as they show higher rates of self-reported burnout, mental disorders, and smoking among psychiatrists when compared to other physicians (3-10), and studies showing more favorable health behaviors among surgeons and pediatricians (7-12). We found that the differences between specialties in our study with the majority of participants being nurses were quite similar to those reported for physicians. Earlier studies of nurses suggest more emotional exhaustion (17) but no excess suicidal behavior among psychiatric healthcare personnel (18). A previous review has suggested high burnout rates among intensive care unit nursing staff (21) while in our study, intensive care personnel had a slightly higher probability of having one indicator of poor health, with outcomespecific results suggesting that this risk factor is smoking rather than mental health problems. However, these comparisons should be interpreted cautiously because many of the earlier studies included professionals from private and public sectors and hospital as well as community settings whereas our study focused on personnel working in public hospitals.

There are at least three explanations for our findings regarding psychiatric staff: (i) greater exposure to occupational health hazards; (ii) health-related selection into the specialty; and (iii) illness behaviors. We found that psychiatric staff reported lower levels of stressful work characteristics, as indicated by "job strain" and "effortreward imbalance", than employees in the somatic disease hospital wards. Psychiatric personnel also reported higher level of organizational justice. These findings are in line with some other studies $(15-17,31)$. However, psychiatric personnel were more frequently exposed to violence at work than employees working in other specialties although adjustment for these work characteristics did not remarkably affect the findings. These findings suggest that the demand-control model (32) and other traditional work stressors may not be as useful in attempts to explain morbidity in human service work. Therefore the research should look into work-related stressors other than the traditional ones as well as resilience factors in future studies.

Other stress-inducing factors in psychiatry may relate to patients' suicide, negative emotions elicited by patients, chronic staff shortages, efficiency issues, restructuring, and other changes in psychiatric health care (1, 2, 33-35) as well as a principal ethical dilemma reported by psychiatrists, that is, under-treatment of patients while somatic specialists tend to report overtreatment (36). It is likely that nursing staff faces the same ethical dilemma.

High emotional load in psychiatry is understandable, because the main "instrument" is interpersonal interaction 
including emotional aspects, and the patients are emotionally challenging. Development of strategies to deal with such patients is essential (2). Using empathy, an essential skill for therapeutic interaction, brings in emotional load [also referred as "compassion fatigue" $(37,38)$ ], controversies, and even secondary traumatization (39). However, distinguishing secondary traumatization of mental health workers from other sources of distress arising within the workplace has been considered difficult (38).

We found the most consistent association of working in psychiatry versus other specialties with mental health outcomes. This supports the hypothesis of greater exposure to work stress as mental disorders have been found to be linked with work stress (40). However, as already discussed, the "traditional" work stress indicators did not explain the difference between psychiatry and other specialties suggesting exposure to work stressors other than those measured in this study. The associations may be complex because, for instance, adjustment for smoking, an independent predictor of depression (41), did not explain the findings either. However, the probability of having a diagnosed chronic physical disease was also more common among psychiatric staff and not all of these outcomes are stress-related.

In the present study context, health-related selection is a process in which health is one of the determinants of what types of jobs people are likely to enter (2, $42,43)$. With regard to psychiatry, specialization and employment in psychiatry may be easier due to reduced competition (psychiatry may be less attractive as a profession than, for example, surgery or pediatrics), which is associated with staff shortages in many psychiatric hospitals. These phenomena may result in selection for health and related attributes such as academic success. Second, some people with pre-existing mental disorders or those with familial predisposition may have a high interest in psychiatry. They may therefore apply to study psychiatry in order to increase understanding of their own mental health problems or those of relatives. We assessed antidepressant use over 11 years among psychiatric and non-psychiatric staff and found that, in the first three years, there was no significant difference in antidepressant use between the specialties while in the following years psychiatric staff had 1.4 to 1.8 -fold higher prevalence of antidepressant use. Gradually increasing differences in antidepressant use between specialties suggest that health selection might not be the primary driver for the excess health problems among psychiatric staff. Indeed, aspects of work along with personal vulnerability may contribute to increased morbidity risk among psychiatric staff (2).

Another issue is whether there has been a reduction in stigma towards antidepressant use among psychiatric personnel during the time when antidepressants became increasingly common and accepted by their patients.
Psychiatric staff might also have better knowledge about the medication and among them, mental disorders may involve less of a stigma. However, our results from a one-year prospective analysis provide no evidence of "unnecessary" anxiolytic and hypnotic use among psychiatric staff.

The specific strengths of our study are its large sample size and prospective study design. Furthermore, we culled objective data from independent national registers, which provide comprehensive records of sickness absences $>9$ days with physician-certified diagnoses and purchases of medication. Unlike earlier studies on the subject, we were thus able to minimize potential biases due to self-report although confounding by unobserved or imprecisely measured variables is still possible.

We used employer's work unit codes to determine specialty at baseline. This may have caused some misclassifications, since part of the personnel may be specialized in domains other than where they currently work, and employees may have changed work units (ie, specialization) during the follow-up. However, we adjusted analyses for type of employment and, in the sensitivity analyses, we restricted the sample to permanent employees who are likely to be specialized to that domain and unlikely to move. However, our study focused on working in a specific medical domain, not about an individual's specialization to a specific medical specialty, as in many earlier studies. Furthermore, some of our results were based on cross-sectional data which limit conclusions about the direction of causality. In addition, this study was carried out in 6 of 21 hospital districts in Finland. Replications in other parts of Finland as well as in countries with different healthcare systems are important.

In conclusion, this study suggests that the prevalence of hospital employees with an adverse health risk profile is higher in psychiatry than other specialties. In contrast, employees working in surgery had the lowest probability for health problems. Our findings emphasize the importance of paying attention to the health of psychiatric staff and the need for preventive programs. Further research with longer follow-up periods is necessary to examine the contribution of work-related stress, health selection, and help-seeking behavior to the observed associations.

\section{Acknowledgements}

This work was supported by the Academy of Finland (grants \#124322, \#124271, \#129262 and \#132944). G David Batty is a Wellcome Trust Fellow and Mika Kivimäki is supported by the BUPA Foundation, UK, and the EU New OSH ERA Research Programme. 


\section{References}

1. Mateen FJ. Health-care worker burnout and the mental health imperative. Lancet. 2009;374:596-7. http://dx.doi. org/10.1016/S0140-6736(09)61483-5.

2. Arnetz BB. Psychosocial challenges facing physicians of today. Soc Sci Med. 2001;52:203-13. http://dx.doi. org/10.1016/S0277-9536(00)00220-3.

3. Hawton K, Clements A, Sakarovitch C, Simkin S, Deeks JJ. Suicide in doctors: a study of risk according to gender, seniority, and specialty in medical practitioners in England and Wales, 1979-1995. J Epidemiol Community Health. 2001;55:296-300. http://dx.doi.org/10.1136/jech.55.5.296.

4 Kumar S. Burnout in psychiatrists. World Psychiatry. 2007;6:186-9.

5. Carpenter LM, Swerdlow AJ, Fear NT. Mortality of doctors in different specialties: findings from a cohort of 20000 NHS hospital consultants. Occup Environ Med. 1997;54:388-95. http://dx.doi.org/10.1136/oem.54.6.388

6. Töyry S, Räsänen K, Kujala S, Aärimaa M, Juntunen J, Kalimo R. Self-reported health, illness, and self-care among Finnish physicians. Arch Fam Med. 2000;9:1079-85. http://dx.doi. org/10.1001/archfami.9.10.1079.

7. Hughes PH, Baldwin DC, Sheehan DV, Conard S, Storr CL. Resident physician substance use, by specialty. Am J Psychiatry. 1992;149:1348-54.

8. Talbott GD, Gallegos KV, Wilson PO, Porter TL. The Medical Association of Georgia's Impaired Physicians Program. Review of the first 1000 physicians: analysis of specialty. JAMA. 1987;257:2927-30. http://dx.doi.org/10.1001/ jama.1987.03390210075028.

9. Frank E, Boswell L, Dickstein LJ, et al. Characteristics of female psychiatrists. Am J Psychiatry. 2001;158:205-12. http://dx.doi.org/10.1176/appi.ajp.158.2.205.

10. Hughes PH, Storr CL, Brandenburg NA, Chapman DP. Physician substance use by medical specialty. J Addict Disord. 1999;18:23-37. http://dx.doi.org/10.1300/J069v18n02_03

11. Arnetz BB, Hörte L-G, Hedberg A, Theorell T, Allander E, Malker H. Läkarens dödlighetsmönster $\mathrm{i}$ ischemisk hjärtsjukdom (IHD) och suicid - data baserade på dödsfallsregistret och socialstyrelsens läkarförteckning [Mortality pattern from ischemic heart disease and suicide: a cohort study]. Stockholm: Karolinska Institute, Stressforskningsrapporter No.185; 1986.

12. Asp S, Hernberg S, Collan Y. Mortality among Finnish doctors, 1953-1972. Scand J Soc Med. 1979;7:55-62.

13. Ramirez AJ, Graham J, Richards MA, Cull A, Gregory WM. Mental health of hospital consultants: the effects of stress and satisfaction at work. Lancet. 1996;347:724-8. http://dx.doi. org/10.1016/S0140-6736(96)90077-X.

14. Juntunen J, Asp S, Olkinuora M, Äärimaa M, Strid L, Kauttu K. Doctors' drinking habits and consumption of alcohol. BMJ. 1988;297:951-4. http://dx.doi.org/10.1136/ bmj.297.6654.951.
15. Plant ML, Plant MA, Foster J. Stress, alcohol, tobacco and illicit drug use amongst nurses: a Scottish study. J Adv Nurs. 1992;17:1057-67. http://dx.doi. org/10.1111/j.1365-2648.1992.tb02040.x.

16. Schulz M, Damkröger A, Heins C, Wehlitz L, Löhr M, Driessen $\mathrm{M}$, et al. Effort-reward imbalance and burnout among German nurses in medical compared with psychiatric hospital settings. J Psychiatr Ment Health Nurs. 2009;16:225-33. http://dx.doi.org/10.1111/j.1365-2850.2008.01355.x.

17. Tummers GER, Janssen PPM, Landeweerd A, Houkes I. A comparative study of work characteristics and reactions between general and mental health nurses: a multi-sample analysis. J Adv Nurs. 2001;36:151-62. http://dx.doi. org/10.1046/j.1365-2648.2001.01952.x.

18. Ramberg IL, Wasserman D, Prevalence of reported suicidal behaviour in the general population and mental healthcare staff. Psychol Med. 2000;30:1189-96.http://dx.doi. org/10.1017/S003329179900238X.

19. Gillespie M, Melby V. Burnout among nursing staff in accident and emergency and acute medicine: a comparative study. J Clin Nurs. 2003;12:842-51. http://dx.doi.org/10.1046/j.13652702.2003.00802.x.

20. Petterson IL, Arnetz BB, Arnetz JE, Hörte LG. Work environment, skills utilization and health of Swedish nurses - results from a national questionnaire study. Psychother Psychosom. 1995;64:20-31. http://dx.doi. org/10.1159/000288987.

21. Embriaco L, Papazian L, Kentish-Barnes N, Pochard F, Azoulay E. Burnout syndrome among critical care healthcare workers. Curr Opin Crit Care. 2007;13:482-8. http://dx.doi. org/10.1097/MCC.0b013e3282efd28a.

22. Virtanen M, Pentti J, Vahtera J, Ferrie J, Stansfeld SA, Helenius $\mathrm{H}$, et al. Overcrowding in hospital wards as a predictor of antidepressant treatment among hospital staff. Am J Psychiatry 2008;165:1482-6. http://dx.doi.org/10.1176/ appi.ajp.2008.07121929.

23. Rimm EB, Williams P, Fosher K, Criqui M, Stampfer MJ. Moderate alcohol intake and lower risk of coronary heart disease: Meta-analysis of effects on lipids and haemostatic factors. BMJ. 1999;319:1523-8. http://dx.doi.org/10.1136/ bmj.319.7224.1523.

24. Kujala UM, Kaprio J, Sarna S, Koskenvuo M. Relationship of leisure-time physical activity and mortality: The Finnish twin cohort. JAMA. 1998;279:440-4. http://dx.doi.org/10.1001/ jama.279.6.440.

25. Karasek R, Theorell T. Healthy work: stress, productivity, and the reconstruction of working life. New York: Basic Books; 1990.

26. Siegrist J. Adverse health effects of high-effort/low-reward conditions. J Occup Health Psychol. 1996;1:27-41. http:// dx.doi.org/10.1037/1076-8998.1.1.27.

27. Moorman RH. Relationship between organizational justice and organizational citizenship behaviors: Do fairness perceptions influence employee citizenship? J Appl Psychol. 1991;76:84555. http://dx.doi.org/10.1037/0021-9010.76.6.845. 
28. Virtanen M, Vahtera J, Batty GD, Tuisku K, Pentti J, Oksanen $\mathrm{T}$, et al. Overcrowding in psychiatric wards and physical assaults on staff: a data-linked longitudinal study. $\mathrm{Br} \mathrm{J}$ Psychiatry. 2011;198:149-55. http://dx.doi.org/10.1192/bjp. bp. 110.082388 .

29. Koskenvuo M, Kaprio J, Rose RJ, Kesäniemi A, Sarna S, Heikkilä K, et al. Hostility as a risk factor for mortality and ischemic heart disease in men. Psychosom Med. 1988;50:330-40.

30. WHO Collaborating Centre for Drug Statistics Methodology. Guidelines for ATC Classification and DDD Assignment. Oslo: WHO Collaborating Centre for Drug Statistics; 2004.

31. Nylenna M, Aasland OG. Tidsskr nor Laegeforen [Job satisfaction among Norwegian doctors]. 2010;130:1028-31. http://dx.doi.org/10.4045/tidsskr.09.0955.

32. Söderfeldt B, Söderfeldt M, Muntaner C, O’Campo P, Warg LE, Ohlson CG. Psychosocial work environment in human service organizations: a conceptual analysis and development of the demand-control model. Soc Sci Med. 1996;42:1217-26. http://dx.doi.org/10.1016/0277-9536(95)00231-6.

33. Katschnig H. Are psychiatrists an endangered species? Observations on internal and external challenges to the profession. World Psychiatry. 2010;9:21-8.

34. Fischer J, Kumar S, Hatcher S. What makes psychiatry such as stressful profession? A qualitative study. Australas Psychiatry. 2007;15:417-21. http://dx.doi. org/10.1080/10398560701439699.

35. Arnetz BB, Lucas T, Arnetz JE. Organizational climate, occupational stress and employee mental health: mediating effects of organizational efficiency. J Occup Environ
Med. 2011;53:34-42. http://dx.doi.org/10.1097/ JOM.0b013e3181ffo5b.

36. Saarni SI, Halila L, Palmu P, Vänskä J. Ethically problematic treatment decisions in different medical specialties. J Med Ethics. 2008;34:262-7. http://dx.doi.org/10.1136/ jme.2007.020529.

37. Coetzee SK, Klopper HC. Compassion fatigue within nursing practice: a concept analysis. Nurs Health Sci. 2010;12:235-43. http://dx.doi.org/10.1111/j.1442-2018.2010.00526.x.

38. Sabin-Farrell R, Turpin G. Vicarious traumatization: implications for the mental health of health workers? Clin Psychol Rev. 2003;3:449-80. http://dx.doi.org/10.1016/ S0272-7358(03)00030-8.

39. Rothschild B. Help for the helper. New York: Norton; 2006.

40. Stansfeld S, Candy B. Psychosocial work environment and mental health - a meta-analysis. Scand J Work Environ Health. 2006;32:443-62.

41. Klungsøyr O, Nygård JF, Sørensen T, Sandanger I. Cigarette smoking and incidence of first depressive episode: an 11year, population-based follow-up study. Am J Epidemiol. 2006;163:421-32. http://dx.doi.org/10.1093/aje/kwj058.

42. Blane DB. An assessment of the Black Report's explanation of health inequalities. Sociol Health Illness. 1985;7:423-45. http://dx.doi.org/10.1111/1467-9566.ep10832355.

43. Fergusson DM, Woodward LJ. Mental health, educational and social role outcomes of adolescents with depression. Arch Gen Psychiatry. 2002;59:225-31. http://dx.doi.org/10.1001/ archpsyc.59.3.225.

Received for publication: 12 August 2011 